Zeledón and there are two chapters on its pathology, one by A. Anselmi and F. Moleiro and the other by F. Köberle.

Most of the chapters are well written and straightforward accounts which reveal both the extent of and gaps in present knowledge. The two chapters on the pathology of Chagas' disease represent alternative views. The section on antigenic variation in trypanosomiasis is probably the most speculative in the symposium and the one least likely to survive the passage of time.

What emerges is an excellent statement of the present situation with regard to these diseases. Much of it is a textbook and will be used as such. It is also a source book for those seeking new (or first) fields to conquer and a reference book for those involved in teaching, research and clinical medicine. Two points, however, may confuse uninitiated readers. Leishmania aethiopica is mentioned on pages 102 and 250 but not in the check list in the first chapter. Although described after the meeting, this parasite should have been added in proof. Schizotrypanum is given subgeneric status in chapter 1 but occasionally reverts to generic status during subsequent discussions.

One fact comes over above all others from this symposium, the deficiency in understanding of Chagas' disease. Here is a disease that affects 35 million people and in parts of Brazil is responsible for one third of all adult deaths. Yet we do not know how many forms of the causative agent, Trypanosoma cruzi, exist nor do we have a single drug that is effective once the parasite reaches its host cell.

F. E. G. Cox

\section{History of salt water}

The Development of the Chlorinity/ Salinity Concept in Oceanography. By William J. Wallace. Pp. xii +227.

Elsevier Oceanography Series, Vol. 7. (Elsevier: Amsterdam, London and New York, 1974.) Dfl.46; \$17.70.

THE author starts with a careful summary of Greek and Arabic writings about salt, mineral waters and seawater. Errors that appear in Riley and Skirrow are repeated in a paragraph quoting a letter from Synesius to Hypatia describing a hydrometer in about 410 AD. Boyle's Observations and Experiments on the Saltness of the Sea is treated as a landmark, and also Halley's study of evaporation, particularly his work on the Mediterranean Sea, and on condensation at higher levels as he saw it in St Helena. Lavoisier is shown as mainly interested in medicinal waters though adding considerably to what was known about the sea. Bergman's analyses began to hint that the main constituents of sea salt are always present in the same proportions.
The careful work of John Murray, published in 1818 , brought reproducibility to sea water analysis. Marcet formulated the principle of constancy of composition in 1819, and Forchhammer, and Dittmar who analysed samples collected during HMS Challenger's circumnavigation, confirmed it. Measurement of total solids by evaporation does not give consistent results, and experience showed that for common use it is better to rely on estimated values based on measurements of chlorine content. Sorensen in Sweden and Knudsen in Denmark put the procedure on a systematic basis by careful work in 1899-1902. They refined the methods, initiated a distribution of Standard Sea Water for everyone to use, and published tables relating chlorinity to salinity and density, so that before long all results could he combined into maps and sections sufficiently detailed and consistent to trace water movements, and to work out pressure patterns. There was no reason other than some self satisfaction or sense of completeness why salinities should have been plotted rather than the chlorinities on which they were based.

The final part of the book deals with changes inspired by more recent improvements. The measurement of electrical conductivity is now an easier and more accurate routine than chlorine determination, and salinity has been newly defined in terms of conductivity. The author reviews the problems that remain. The Standard Sea Water is being used as a reference for conductivity instead of chlorinity, though each main batch is checked. The difficulties of measuring absolute conductivity and density are still not surmounted. So long as careful checks are maintained the standards are adequate, but the author thinks we may lose if unquestioning acceptance of constant relationships between chlorinity, electrical conductivity, density, and other physical properties and chemical constituents, discourages critical study of small exceptions to the rule. Such exceptions may, as refined methods are more widely used, provide new lines of investigation into the origin and history of water mixtures.

The author has done a great service to the history of marine chemistry. Judging from his text and very extensive bibliography his work seems to have been completed in about 1970, or he would have included something about Kremling's (1971) new method of measuring density, and recent reports of the Joint Panel on Oceanographic Tables and Standands, set up by Unesco and the international marine science organisations to take care of the new problems.

G. E. R. Deacon

\section{Abandon dualism}

Quantum Mechanics in a New Key. By Alfred Landé. Pp. $x+130$. (Exposition: New York, 1973) $\$ 6.50$.

THE author states in the introduction his intention to derive quantum mechanics from three non-quantal postulates. These are: symmetry (the probability $P_{\beta \alpha}$ connecting two states $\alpha$ and $\beta$ is twoway symmetric, so that $P_{\alpha \beta}=P_{\beta \alpha}$ ); correspondence (the probabilities connecting various pairs of states are to be interdependent by way of a general theorem which is to yield the ordinary probability addition law $P_{\alpha \gamma}=\Sigma_{\beta} P_{\alpha \beta} P_{\beta \gamma}$ in the average; and covariance (there are no preferred zero points for energy and momentum, nor for time and space coordinates). In this way the author hopes to demonstrate that quantum mechanics is a logically consistent theory of probability and symmetry, and that the waveparticle dualism which emerged in the early history of the subject may now be abandoned as an unnecessary mystification.

In chapter 1 he discusses several critical experiments, and uses Duane's theory of diffraction, originally put forward to explain the diffraction of photons, to show how wavelike effects such as interference may be explained without assuming the existence of matter waves. In chapters 2 and 3 the first and second postulates are introduced and their consequences are considered; these are further developed in chapter 4 . The third postulate and its consequences are discussed in chapter 5. Statistical thermodynamics is briefly considered in chapter 6; the author returns to his main theme of observation and interpretation in chapter 7 .

The aims of the book are admirable, and most physicists would probably accept the philosophy which it expounds. The lack of mathematical rigour would not, in itself, be a disadvantage in a book of this kind, but unfortunately the author's conclusions do not always seem to follow from his postulates. For example, the statements $P_{\alpha \beta}=\left|\Psi_{\alpha \beta}\right|^{2}$ where $\Psi_{\alpha \beta}=\Psi_{\beta \alpha}^{*} \quad$ and $\quad \Psi_{\alpha \gamma}=\Sigma_{\beta} \Psi_{\alpha \beta} \Psi_{\beta \gamma} \quad$ do not obviously follow from the first two postulates. Rather these should themselves be regarded as the basic postulates, from which the first two postulates are then easily deduced. Symmetry and correspondence provide a guide to the choice of postulates, rather than themselves being postulates. The deduction of the result $\Psi_{p q}=C \exp (2 i \pi p q / h)$ in chapter 5 from the postulate of covariance, and the derivation of the Schrodinger wave equation from this result are elegant, but I think that the reader would have been helped by a more detailed explanation of the mathematical reasoning behind the steps of the arguments.

J. E. G. FARINA 